\title{
INTENTO SUICIDA Y PSICOSIS DEBIDO A DÉFICIT DE VITAMINA B12 A PROPÓSITO DE UN CASO
}

\author{
Suicide attempt and psychosis due to deficit of vitamin B12, for the purpose of a case
}

\section{Zuleika Morillo, MD, Deseado S. Guzmán y Yosira A. Ortiz}

Recibido: 6 marzo 2018 Aprobado: 24 mayo 2018

Cómo citar: Morillo de Nieto Z, Guzmán Esquea D, Ortiz Y. Intento suicida y psicosis debida a déficit de vitamina B12, a propósito de un caso. Ciencia Y Salud [Internet]. 14sep.2018 [citado 14 sep.2018];2(3):55-1. Available from: https://revistas.intec. edu.do/index.php/cisa/article/view/1286

\section{Resumen}

Introducción: El folato y la vitamina B12 juegan un papel importante en el correcto desarrollo, diferenciación y funcionamiento del Sistema Nervioso Central. Intervienen en la producción de mielina y son indispensables para numerosas reacciones que implican la metilación y biosíntesis de ácidos nucleicos, además de intervenir en la síntesis de proteínas, neurotransmisores, ácidos grasos y fosfolípidos. Su deficiencia se asocia a alteraciones cognitivas, irritabilidad, depresión, psicosis, demencia, entre otras.

Material y Métodos: En este trabajo se presenta el caso de una joven de 17 ańos, sin antecedentes mórbidos conocidos, que presentó cuadro de un mes de evolución, caracterizado por intento suicida, alucinaciones visuales y auditivas, idea delirante de daño tipo persecutoria, cefalea y parestesias en miembros inferiores. Ingresada en

Clínica Infantil Dr. Robert Reid Cabral

Santo Domingo, República Dominicana

Dra. Zuleika Morillo de Nieto, MD

Paidoszu@hotmail.com

Dr. Deseado Guzmán Esquea

Deseado@gmail.com

Dra. Yosira Ortiz

draortizdelacruz@gmail.com unidad de psiquiatría y consultada por departamento de neurología.

Se le realizaron analíticas y estudios especializados que incluían hemograma, glicemia, perfil renal, hepático, virales, electrolitos, perfil tiroideo, panel 6, tomografía de cráneo y EEG, sin evidencia patológica. Posteriormente con vitamina B12 y ácido fólico, evidenciando niveles de Vit B12 reducidos (110pg-ml), iniciamos la reposición de vitamina B12 intramuscular y ańadimos Risperidona 3 mg cada 24 horas.

Conclusión: La paciente presentó remisión total de los síntomas, a partir del tratamiento de Vitamina B12 y los controles pertinentes del departamento de psiquiatría y hematología.

Palabras Claves: Intento suicida, psicosis, parestesias, déficit de B12.

\section{Summary}

Introduction: Folate and vitamin B12 play an important role in the proper development, differentiation and functioning of the CNS. They intervene in the production of myelin, and are indispensable for numerous reactions involving the methylation and biosynthesis of nucleic acids. In addition to intervening in the synthesis of proteins, neurotransmitters, fatty acids and phospholipids. Its 
deficiency is associated with cognitive alterations, irritability, depression, psychosis, dementia,among others.

Material and Methods: We present the case of a 17-yearold girl, with no known morbid history, who presented a month evolution, characterized by suicidal attempt, visual and auditory hallucinations, delusional idea of persecutory type damage, headache and paresthesias in lower limbs. Entered psychiatry unit and interconsultated with department of neurology.

Analyzes and specialized studies were carried out that included hemogram, glycemia, renal, hepatic, viral, electrolyte, thyroid profile, panel 6, tomography of the skull and EEG, without pathological evidence. Subsequently, Vitamin B12 and folic acid were performed, evidencing reduced B12 Vit levels (110pg-ml), we started the replacement of intramuscular vitamin B12 and added Risperidone $3 \mathrm{mg}$ every 24 hours.

Conclusion: The patient presented total remission of symptoms. She is currently asymptomatic with Vitamin B12 maintenance treatment and controls by department of psychiatry and hematology.

Keywords: Suicidal attempt, psychosis, paresthesias, B12 deficit.

\section{Introducción}

Los estudios de laboratorio proporcionan una base firme para los cambios patológicos en el Sistema Nervioso Central (SNC), como resultado de la privación de vitamina B. Se sabe que muchos componentes del complejo B juegan un papel esencial en los procesos metabólicos dentro del cerebro, como son: el pirofosfato de tiamina, una coenzima involucrada en el metabolismo de carbohidratos, particularmente en la oxidación de piruvato, que también puede ser necesario para la adecuada transmisión de impulsos nerviosos; el ácido nicotínico, actúa como parte constituyente de las coenzimas necesarias para el metabolismo de la glucosa; la riboflavina, esta actúa de manera similar al ácido nioctínico; el ácido pantoténico, este es necesario para la formación de acetilcolina; y la piridoxina, que se convierte en fosfato de piridoxal, una coenzima fundamental para varios sistemas enzimáticos interesados en procesos metabólicos cerebrales.

La vitamina B12 y el ácido fólico cumplen un rol importante en el correcto desarrollo, diferenciación y funcionamiento del SNC. Intervienen en la producción de mielina y son indispensables para numerosas reacciones que implican la metilación y biosíntesis de ácidos nucleicos, además de intervenir en la síntesis de proteínas, neurotransmisores, hormonas, ácidos grasos y fosfolípidos. Su deficiencia se asocia a alteraciones cognitivas, astenia, letargo, irritabilidad, depresión, psicosis, síntomas ansiosos, trastorno obsesivo compulsivo, demencia, entre otras ${ }^{1,2}$.

Si hablamos dehistoria, no fue sino hasta la década de 1930 quela significación completa dela deficiencia de vitamina $B$, en relación con el trastorno psiquiátrico comenzó a ser apreciado, aunque el trastorno mental había sido reconocido como una parte integral del síndrome de la pelagra, desde sus primeras descripciones. En la década de 1930, sin embargo, se identificaron varios componentes del complejo B, y la observación cuidadosa pronto extendió la conciencia de sus funciones. Los estudios experimentales mostraron que la privación podría conducir a síntomas psiquiátricos incluso antes que las manifestaciones en otros sistemas aparecieran.

\section{Caso Clínico}

Presentamos el caso de una paciente de 17 años, soltera, sin antecedentes mórbidos conocidos, sin historia psiquiátrica anterior, historia psiquiátrica familiar negada. La misma presentó un intento de suicidio frustrado (por ahorcamiento), por lo que fue llevada al hospital de atención primaria, donde permanece por 5 días en observación, y luego referida al hospital de atención terciaria, para ser evaluada por el departamento de medicina interna y psiquiatría. 
El examen físico no reveló hallazgos relevantes. En el interrogatorio con los familiares, los mismos refieren que la joven presenta un cuadro de 1 mes de evolución, caracterizado por cefaleas de intensidad moderada y parestesias en miembros inferiores y superiores. Afirman que la ven temerosa y presenta dificultad para conciliar el sueño, pasando a dormir con sus padres en las últimas dos semanas, en ocasiones la ven mirando fijo las paredes de su cuarto, pero al cuestionarla, esta se niega a dar explicaciones. En el examen mental de la joven nos encontramos un lenguaje en tono bajo, curso lento, discurso coherente que se torna circunstancial, ánimo eutímico, afecto incongruente (suspicaz) quien admite: síntomas ansiosos (inquietud, sudoraciones, cefalea), alteración del patrón sueño (insomnio), idea delirante de daño tipo persecutoria, alteraciones perceptivas tipo alucinaciones visuales y auditivas, intento suicida en pasado. Sin embargo, la paciente niega demás síntomas afectivos, psicóticos y ansiosos, con ligera alteración de la memoria y cognición, con crítica de enfermedad mental.

Ante tal situación decidimos ingresar la paciente con diagnóstico de intento suicida y trastorno psicótico a especificar, probable, debido a enfermedad médica. En el ingreso se realiza hemograma, glicemia, perfil tiroideo, renal y hepático, pruebas virales, electrolitos, tomografía de cráneo, electrocardiograma y radiografía de tórax. Todas estas pruebas se encontraban dentro de parámetros de normalidad. Decidimos consultar al departamento de neurología, quienes recomiendan resonancia magnética craneal, determinaciones séricas de vitamina B12 y ácido fólico, y electroencefalograma de 60 minutos en vigilia y sueño.

En el internamiento, la paciente inicia tratamiento con antipsicótico Risperidona $3 \mathrm{mg}$, cada 24 horas, vía oral, mostrando leve mejoría de síntomas psiquiátricos. La resonancia magnética craneal y el electroencefalograma muestran resultados dentro de parámetros de normalidad, mientras que recibimos valores de vitamina $\mathrm{B} 12 \mathrm{de} 110 \mathrm{pg} / \mathrm{dl}$
(200-500) y ácido fólico dentro de la normalidad $(15 \mathrm{np} / \mathrm{dl})$.

En este momento se interconsulta al departamento de hematología, que inicia un tratamiento con reposición de vitamina B12. La paciente fue tratada con hidroxocobalamina intramuscular 1000 $\mu \mathrm{g}$, durante 7 días, luego pasó a una dosis semanal, durante 6 semanas, y a partir de entonces cada tres meses hasta la actualidad; mostrando mejoría de síntomas de enfermedad mental, y egresada 3 semanas más tarde. En la actualidad se encuentra en remisión de síntomas mentales, y asiste a seguimientos regulares de psiquiatría y hematología.

El diagnóstico fue un trastorno psicótico debido a déficit de vitamina B12. Este diagnóstico, aunque es bien conocido, hasta la fecha no existen casos reportados en nuestro país.

\section{Discusión}

En el caso presentado nos encontramos ante una joven con un cuadro de un mes de evolución caracterizado por cefalea, parestesias de miembros inferiores y síntomas psicóticos, sin antecedentes psiquiátricos conocidos. Con esta sintomatología nos planteamos un probable origen neurológico y luego de realizar las analíticas de lugar, encontramos un déficit de vitamina B12 importante, sin haber presentado anemia en el hemograma; que al ser repuesto con hidroxicobalamina intramuscular, mejoró totalmente la sintomatología neuropsiquiátrica.

El déficit de vitamina B12 es usualmente el resultado de una inadecuada absorción, como en la anemia perniciosa, enfermedades gástricas, o a un inadecuado aporte. Es conocida por sus consecuencias tanto hematológicas como neuropsiquiátricas. Aunque muchos de los casos las alteraciones psiquiátricas ocurren junto con anemia macrocítica, las alteraciones neuropsiquiátricas pueden ocurrir en ausencia de alteraciones hematológicas. 


\section{Figura 1. Metabolismo de la Homocisteína}

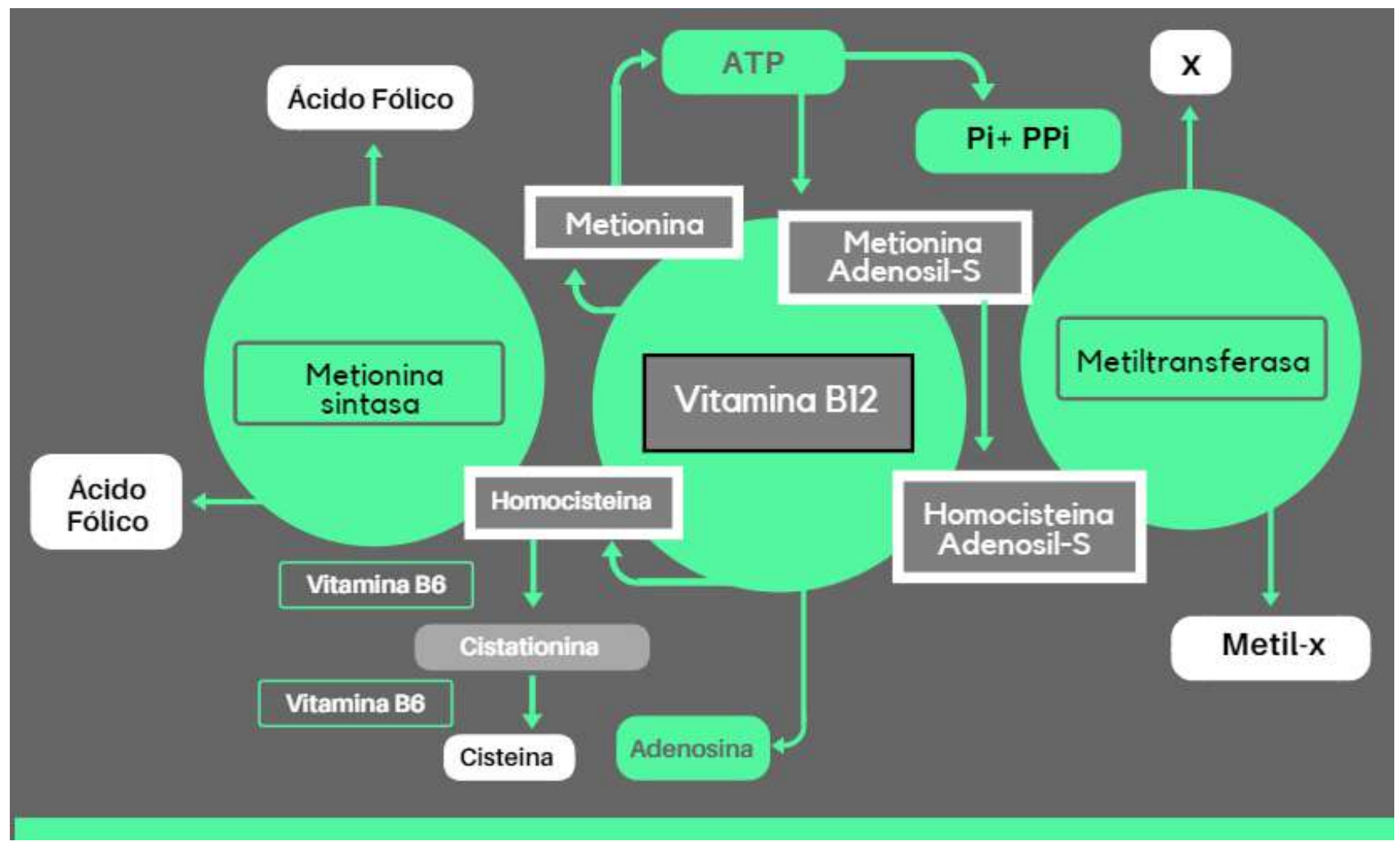

Actualmente, se vincula el ser homocigoto para la metiltetrahidrofolato del gen $\mathrm{c} 677 \mathrm{~T}$ de la reductasa ( MTHFR) como una probable causa de que personas con deficiencias de vitamina B12 desarrollen primero síntomas psiquiátricos, mientras otros desarrollan inicio de anemia megaloblástica. ${ }^{3}$ La explicación planteada es que las personas homocigotas para este alelo presentan cierta protección para presentar la anemia, y esta alteración podría producir una disociación entre la enfermedad hematológica y neurológica. En el caso de nuestra paciente la misma no presentaba alteraciones hematológicas en el hemograma, pero sí varios síntomas neuropsiquiátricos.

Los síntomas neuropsiquiátricos de la deficiencia de vitamina B12, generalmente comienzan con parestesias en pies y dedos, debido a la neuropatía periférica precoz, junto con las alteraciones en el sentido vibratorio y la propiocepción. Si no se trata, el trastorno progresa a ataxia espástica, derivada de la desmielinización. La somnolencia es común, además del letargo, demencia, crisis convulsivas y trastornos del estado de ánimo. Así mismo, síntomas psicóticos, caracterizados frecuentemente por ideas delirantes de daño, que en la antigüedad se llamaba Locura Megaloblástica ${ }^{4-6}$.

Otras entidades como el Trastorno Obsesivo Compulsivo, trastornos de ansiedad, la esclerosis múltiple y el Alzheimer se vinculan con la deficiencia de Vitamina B12 y de folato, coincidiendo los autores en un factor común: la hiperhomocisteinemia ${ }^{7,8,9}$.

La homocisteína es un tóxico a nivel neurológico y vascular con múltiples mecanismos, como son el estrés oxidativo y el incremento de la toxicidad de la proteína B-amiloide. Al ser este un compuesto tóxico para el organismo, debe ser metabolizado, y esto puede ser de dos formas, la primera, al ser 
Figura 2. Causas de Hiperhomocisteinemia y sus implicaciones a nivel cerebral

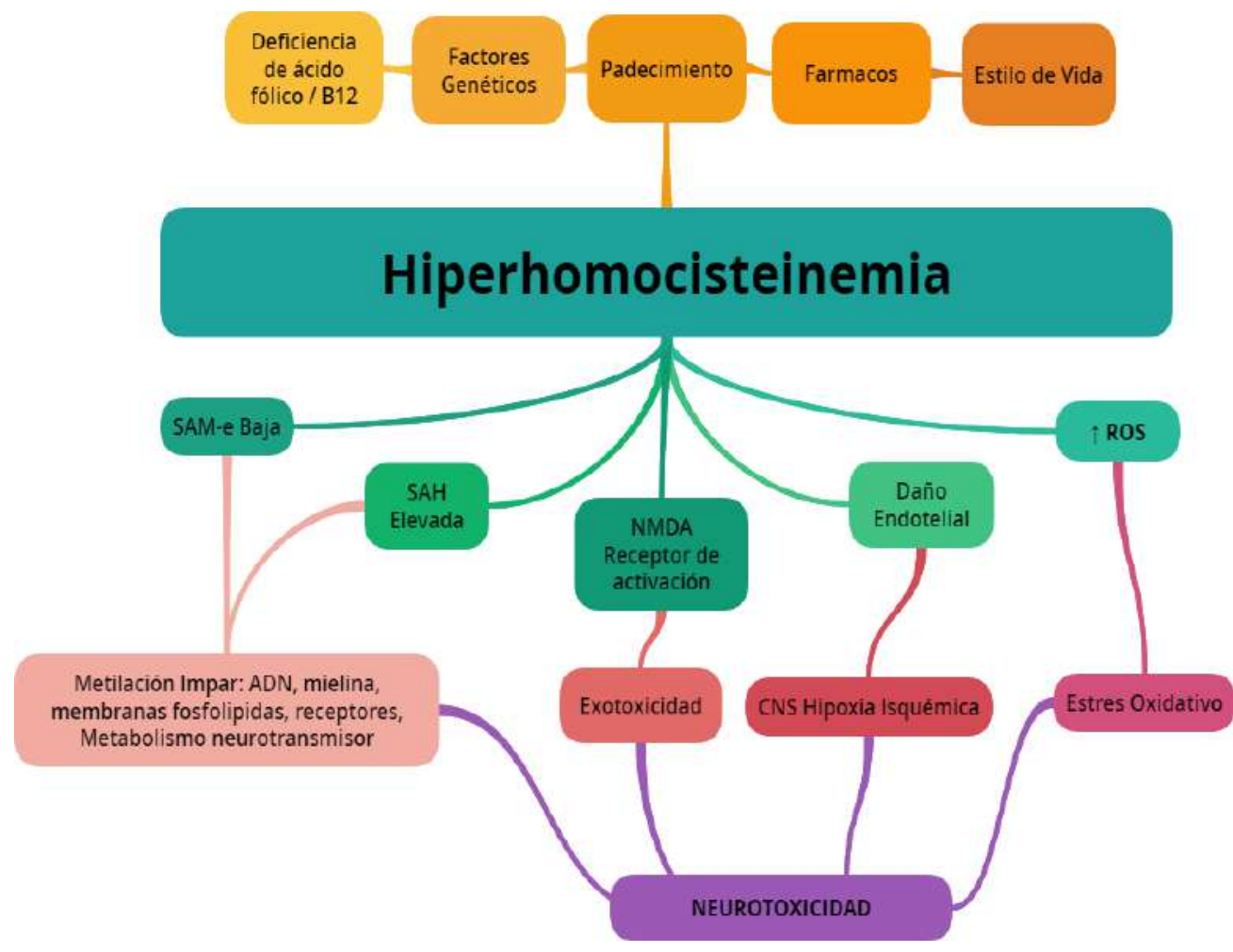

transulfurada a cistatione y subsecuentemente a cisteína, que es un compuesto del glutatión; este proceso depende de la vitamina B6. La otra forma es la remetilación a metionina, por la adhesión de un grupo metilo del 5 - metiltetrahidrofolato (5-MTHF). Esta remetilación puede ser catalizada por la metionina sintetasa, que requiere la vitamina B12 como cofactor en la forma de metilcobalamina ${ }^{10,11,12}$.

Así, un déficit de vitamina B12 o folato puede elevar los niveles de homocisteína. ${ }^{13} \mathrm{~A}$ la vez, esto afectará la síntesis de metionina y de su compuesto activo $S$ adenosilmetionina (SAM), el cual es indispensable para reacciones de metilación, por ejemplo síntesis de proteínas, neurotransmisores, ácidos grasos, fosfolípidos y la metilación del ADN (uno de los mecanismos epigenéticos fundamentales, además de la modificación de histonas y la microdeleción de la cromatina) ${ }^{15}$.
Múltiples mecanismos se relacionan con el aumento de homocisteína, uno de ellos es el déficit de vitamina B12 y el ácido fólico, la alteración del alelo MTHFR del gen C677T, enfermedades como el hipotiroidismo y la enfermedad renal, numerosos fármacos como la L-dopa y el consumo de alcohol, cigarrillo y café.

La homocisteína es tóxico y produce múltiples alteraciones neuropsiquiátricas por diversas vías, aumenta los radicales libres a nivel cerebral, produce dańo endotelial, activa el receptor NMDA de glutamato y altera el funcionamiento y metabolismo de SAM, con sus respectivas consecuencias ya mencionadas anteriormente.

(SAM) S- adenosiltransferasa, (SAH) S-adenosilhomocisteina, (ROS): radicales libres. 


\section{Conclusiones}

Como podemos apreciar son muy diversas las alteraciones asociadas a la deficiencia de vitamina B12. Cada día estamos más cerca de entender con exactitud el porqué de cada síntoma psiquiátrico. Aunque en la actualidad las determinaciones de los niveles séricos de vitamina $\mathrm{B} 12$ y el ácido fólico no forman parte de nuestro protocolo de rutina a la hora de descartar enfermedad médica en nuestros pacientes psiquiátricos; es evidente que se necesita particularizar cada caso, y ante la sospecha, realizar los estudios pertinentes.

\section{Recomendaciones}

Ante pacientes con alteraciones neuropsiquiátricas es necesario incluir dentro del protocolo de analíticas pertinentes, niveles de ácido fólico y vitamina B12, ya que estas deficiencias se vinculan a una extensa gama de psicopatología psiquiátricas.

\section{Referencias Bibliográficas}

1. Karen EC, Renata HB, Wenyang H, Liyuan $\mathrm{D}$, Olga V, Erland A, et al. Low Dietary Folate Interactswith MTHFD1SynthetaseDeficiency in Mice, a Model for the R653Q Variant, to Increase Incidence of Developmental Delays and Defects. The Journal of Nutrition 2018 apr;148(4):501-509. Disponible en: https:// doi.org/10.1093/jn/nxy013

2. Bottiglieri T, Massoumi L, Carpenter LL, Lavretsky H, Mischoulon D. S-Adenosylmethionine (SAMe) for Neuropsychiatric Disorders: A ClinicianOriented Review of Research. The Journal of Clinical Psychiatry. 2017;78(6):e656-e667. Disponible en: http://doi.org/10.4088/ JCP.16r11113.

3. Christensen KE, Bahous RH, Hou W, Deng L, Malysheva O, Arning E, et al. Low
Dietary Folate Interacts with MTHFD1 Synthetase Deficiency in Mice, a Model for the R653Q Variant, to Increase Incidence of Developmental Delays and Defects. The Journal of Nutrition. 2018 apr;148(4):501509. Disponible en https://doi.org/10.1093/ jn/nxy013

4. Paredes MM. Hematological and neurological compromise due to vitamin B12 deficit in infant of a vegetarian mother: case report. Rev. chil. pediatr. [Internet]. 2014 June [cited 2018 Apr 30]; 85(3):337343. Diponible en: https://scielo.conicyt.cl/scielo.php?script=sci_arttext \&pi$\mathrm{d}=$ S0370-41062014000300010\&lng=en.

5. Jatinder SG, FRCPC, Sukhjot Kaur, Bharat M, Neurology of Nutritional Vitamin B12 Deficiency in Infants: Case Series from India and Literature Review, Journal of Child Neurology. 2015;30(13):1831-1837.

6. Brito A, Hertrampf E, Olivares M, Gaitán $D$, Sánchez $H$, Allen Lindsay $H$, et al. Folatos y vitamina B12 en la salud humana. Rev. méd. Chile [Internet]. 2012 Nov [citado 2018 Abr 30]; 140(11):1464-1475. Disponible en: https://scielo.conicyt.cl/ scielo.php?script=sci_arttext\&pid=S0034$98872012001100014 \& \operatorname{lng}=$ es.

7. Forrellat BM, Gómis HI, Gautier du Défaix GH. Vitamina B12: metabolismo y aspectos clínicos de su deficiencia. Rev Cubana Hematol Inmunol Hemoter [Internet]. 1999 Dic [citado 2018 Abr 30]; 15(3):159-174. Disponible en: http://scielo.sld.cu/scielo.php?script=sci_arttext\&pid=S0864-02891999000300001\&ln$\mathrm{g}=\mathrm{es}$.

8. Forrellat Barrios M, Gómis Hernández I, Gautier du Défaix Gómez Hortensia. Vitamina B12: metabolismo y aspectos clínicos de su deficiencia. Rev Cubana Hematol 
Inmunol Hemoter [Internet]. 1999 Dic [citado2018Abr30];15(3):159-174. Disponibleen: http://scielo.sld.cu/scielo.php?script=sci_arttext\&pid=S0864-02891999000300001\&ln$\mathrm{g}=\mathrm{es}$.

9. Behrens MIl, Díaz Va, Vásquez C, Donoso A.Demencia por déficit de vitamina B12: Caso clínico. Rev. méd. Chile [Internet]. 2003 Ago [citado 2018 Abr 30]; 131(8):909914. Disponible en: https://scielo.conicyt.cl/scielo.php?script=sci_arttext \&pi$\mathrm{d}=$ S0034-98872003000800012\&lng=es.

10. Sánchez H, Masferrer D, Lera L, Arancibia E, Ángel B, Albala C. Déficit de vitamina B12 asociado con altas dosis de metaformina en adultos mayores diabéticos. Nutr. Hosp. [Internet]. 2014 Jun [citado 2018 Mayo 01]; 29(6):1394-1400. Disponible en: http:// scielo.isciii.es/scielo.php?script=sci_arttext\&pid=S0212-16112014000600024\&lng=es.

11. Mendoza BermúdezC, Dela Espriella Perdomo Ma. Manifestaciones neuropsiquiátricas del déficit de vitamina B12. rev.colomb.psiquiatr. [Internet]. 2008 Mar [cited $2018 \mathrm{Apr}$ 30]; 37(1):128-138. Disponible en: http:// www.scielo.org.co/scielo.php?script=sci_arttext\&pid=S0034-74502008000100011\&ln$\mathrm{g}=\mathrm{en}$.
12. Malouf R, Areosa Sastre A. Vitamin B12 for cognition. Cochrane Database of Systematic Reviews. 2003;3:CD004394. doi: 10.1002/14651858.CD004394

13. Malouf R, Grimley EJ. Folic acid with or without vitamin B12 for the prevention and treatment of healthy elderly and demented people. Cochrane Database of Systematic Reviews. 2008;4:CD004514. doi: 10.1002/14651858. CD004514.pub2

14. Martí-Carvajal AJ, Solà I, Lathyris D, Dayer M. Homocysteine-lowering interventions for preventing cardiovascular events. Cochrane Database of Systematic Reviews. 2017;8 CD00(6612). doi: 10.1002/14651858. CD006612.pub5.

15. Taylor MJ, Carney SM, Geddes J, Goodwin G. Folate for depressive disorders. Cochrane Database of Systematic Reviews. 2003;2:CD003390. doi: 10.1002/14651858. CD003390.

16. Strobbe, S., \& Van Der Straeten, D. Toward Eradication of B-Vitamin Deficiencies: Considerations for Crop Biofortification. Frontiers in Plant Science. 2018;9(443). Disponible en http://doi.org/10.3389/ fpls.2018.00443 